Discéssion.

Dr. J. C. AUB: Throughout the evening. a discussion of the interrelation of glands of internal secretion has been avoided. The impression from the symposium has been discouraging as to the present value of the use of these various glands in treatment of true endocrine disorders. I think it ought to be brought out that there is the added difticulty of diagnosis, which is increased because most cases present not a defect of one gland but of several.

The basal metabolism, which is the only good functional test for the internal secretions, clemonstrates this difficulty. It is affected not only by the thyroid gland, but also somewhat by disturbances of the gonads and pituitury, though very possibly these last two produce the effect by means of an interrelation with the thyroid. On the other hand, the adrenal gland seems to he an independent mechanism and may cause fluctuations of short duration, probably through its secretion-adrenine. This, therefore, sugrests the complexity of their activities, and the difficulty of determining which gland is primarily at fault in disease. While the relationship of these glands to each other is not clearly understood, we camnot give adequate treatment.

Dr. W. B. Caxion: Dr. Aub asked about the importance of knowing the interrelations of these glands. I have no doubt whatever that they are very intimately interrelated. We have good evidence in some instances of the way in which the destruction, or overaction of one sland will affect another but the relations are highly complex. We must learn about them, however, before we shall have a thoroughly rational mode of taking care of complicated endocrine disorders.

Dr. Lawrence spoke of using the whole adrenal gland. It is a double gland and he is, therefore, using two substances. I suggest he be lold and separate the double gland into its parts and see what each one will do by itself. Then we would know from what he gets his results. I would make the same suggestion to Dr. Ohler. $\mathrm{He}$ is using the whole pituitary. There are, perhaps, three glands, certainly two, in the pituitary, and it might be well for him to try using each part.

\section{(Brininal Artirleg.}

\section{EPIDERMOPHYTOSIS.*}

By Arth ur M. Graen wood, M.D., Boston, Assistant Dermatologist, Massachusetts General Hospital.

THE frequent references to epidermophytosis which we are now seeing and its great prevalence, I believe, warrant this brief sketch of the disease and of its laboratory investigation. The condition is undoubtedly frequently seen by the general practitioner and usually classed with the eczemas, the fact not being recognized that it is parasitic and contagious, extremely difficult to eradicate, and always a potential source of infection to others, besides being exceedingly uncomfortable and often crippling to its possessor.

Epidermophytosis is a form of ringworm. The disease ringworm or tinea includes ringworm of the scalp, tinea capitis; ringworm of the beard, tinea sycosis; tinea circinata, a

- Read in part before the Worcester District Medical Society. superficial, ephemeral ringworm of the glabrous skin; ringworm of the nails; and epidermophytosis, or eczematoid ringworm, and in discussing the last we exclude from consideration all the others.

The fungi causing these various forms of the disease belong to three genera: Microsporon, Trichophyton, and Epidermophyton. Strictly speaking, the name epidermophytosis is not correct, implying as it does that this form of the disease is always caused by some species of the Epidermophyton. That this genus was always the cause was the original conception, but recent work has shown the genus Trichophyton in a large proportion of the cases. However, the name epidermophytosis has thus far been retained since it embodies our concept of the disease as distin. guished from the other forms of ringworm. This concept is as follows: A chronic, contagious, fungous disease of the skin; highly resistant to treatment; at times to be seen on practically any part of the body, but involving most commonly the hands, feet, groins, and peri-anal region, less often the axillae and under the breasts, all locations where there are heat and moisture, factors favorable to the growth of the fungus. Subjectively, it is usually pruritic, at times excessively so, but practically never negligible from the patient's point of view if the process is at all active.

The form of the disease known as tinea cruris or eczema marginatum was described as early as 1869 by Hebra, but the other forms are of much more recent recognition. The following is a very brief clinical description of the more common forms of the disease.

On the feet it ordinarily manifests itself as a maceration of the skin at the depths of the interdigital spaces, most often between the little and fourth toes. At times macerated hyperkeratotic masses ean be shelled out. the so-called soft corns. From the interdigital spaces there may be extensions onto the plantar and dorsal surfaces of the feet, made up of deep resicles with steel-blue centers and collarettes of fine scales where these vesicles have broken and dried. Very commonly the nail of the little toe is infected and is opaque softer than normal. thick and irregular, and other nails mas be involved. This form ordinarily gives rise subjectivelr only to occasional itching, but in warm weather or after much walking in shoes and socks which do not allow sufficient aeration the fungus becomes active, the resicles break out, sometimes becoming bullous on the balls of the toes. and there is severe itching. Another form found on the feet is the hyperkeratotic. in which are seen peculiar yellowish keratotic areas on the soles or heels, not necessarily at points of pressure The form of the disease known as tinea cru 
ris or eczema marginatum is undoubtedly/portant, each one a potential source of infecfamiliar to most physicians. It occurs on the tion. Nor is this a disease of the unwashed inner surfaces of the thighs in the crural re-poutpatient. One of the staff at the Massachugion and may extend downward or onto the setts General Irospital finds it fourth in freserotum and penis and backward over the quency among the diseases he sees, being outperineum to the peri-anal region. It manifests itself as a rather dark red, circular area whose border usually shows many fine vesicles, and whose renter does not clear as does the ordinary tinea circinata. The surface is rather moist and papular and shows delicate scaling. In the perianal region there is usually some whitish, sodden, macerated epithelium to be seen on a reddened base. Subjectively there is a stinging, burning sensation rather than a real itching, but in the peri-anal region there may be a marked pruritus.

The disease, as it affects the hands, eonsists of persistent and recurring attacks, lasting over many years, of areas of erythena will scaling, infiltration and fissuring, and always at some time showing the characteristic deep vesicles, usually at the advancing margins. Its most common location is on the backs of the hands, extending onto the webs of one or several fingers. There is involvement of the contiguous finger surfaces, oftenest of the proximal phalanges, but at times extending to the tips and involving the nails. The so-called washerwoman's eczema is probably a fungus infection, and recent work has shown certain yeasts to be the infecting organism in some cases rather than the genera with which we are eoncerned. ${ }^{1}$ All these fungous infections of the hands have for years been diagnosed as eczema, and we are sceing in our clinies cases which years ago were so considered, which have been relieved, but which recur year after: year. It is not improbable that all cases of eczema of the hands may be due to fungi, excepting dermatitis from external irritants, infuntile eczema, and those of pyogenic origin.

These are the chief and most common forms of epidermophytosis. On other parts of the body they are less distinctive and usually a clue to their origin is to be found in a primary, typical focus on one of the commonly involved regions:

The disease is astonishingly prevalent and apparently increasingly so. In 1919, at the Skin Department of the Massachusetts General Hospital, there were diagnosed 55 cases, including those of eczema marginatum, out of a total of 4065 new patients. At present we see each day at least one new case, frequently four or five, and it should be borne in mind that these are only the ones which are giving symptoms sufficient to bring the patient to the hospital. In a series of 50 patients who came with other troubles one out of two showed evidence of the disease, ready to light up, given the proper conditions, and, what is most im-

numbered only by acne, eczema and alopecia. It is very prevalent among students and gymnasium frequenters, one of the popular narnes for eczema marginatum being "jock strap itch," undoubtedly indicating an accurate observation as to one source of infection. In these gymmasium cases it seems probable that the mats and floors of the showers may be sources of infeetion. In some cases laundries seem to be implieated, and we have several times seen an acute attack follow the doing of a washing in which presumably some of the clothes were infected. Reently we have seen a very acute ease which came on immediately after wearing an old pair of gloves while covering with asbestos preparation a hot steam boiler. The eombination of wearing the probably infeeted gloves with the heat and moisture ineident to the work brought on the sudden, universal infection of both hands. It is quite possible that the fashion of wearing heavy wool socks in recent years is responsible for part of the increase in this disease, since such hosiery not only keeps the feet moist and warm but also camnot be sterilized by boiling.

\section{LABORATORY INVESTIGATION.}

The cultivation and identification of parasitic fungi, or even their microscopic discovery in the seales and hairs, is always a matter involving time and patience. For some reason the usual clinical manifestations of these myeotic affections are out of all proportion to the amount of fungous growth found, and it very often is a matter of long and careful search before the parasite is discovered. Naturally a negative result in such cases leaves one uneertain whether the fungus is really missing or only missed.

It is possible that much of the clinical manifestation of these diseases is in the nature of a trichophytide, which (like the tuberculide) may be a manifestation in a sensitized skin of the presence of the fungus elsewhere on the body. An interesting discussion of the trichophytide is to be found in Bloch's article. ${ }^{2}$ The laboratory end of the study of these mycoses resolves itself into two parts: (a) The examination of the fresh material-scales, hair, or nails; and (b) the cultivation and identification of the fungi.

Examination of the Fresh Material.-(1) Selection of Material. (a) In the macerated interdigital foot cases the best material will be found at the advancing borders of the lesion. In practically every case there will be seen at the junction of the eroded and macerated area 
with the normal skin a delicate sealy border, with the unattached end of the desquamating epidermis toward the lesion. If this elge is raised with forceps and gently pecled off toward the normal skin it will most often be found to contain the parasite. The white, macerated material, in my experience, is useless for microscopical and cultural purposes since it is loaded with contaminating pus organisms and saprophytic fungi. These directions also apply to the cases of tinea cruris or ečema marginatum.

(b) The vesicular type. If seen very early in the vesicular outbreak, one should cut off the roofs of some of the vesicles and invert them on the slide so as to examine their under surface. It is a peculiar fact that not all the vesicles so examined will show the parasites, no matter how carefully the examination is made. As far as I know, there is as yet no way of telling from their clinical appearance which vesicles are infected. It is possible that only the carly lesions will be positive. Only within the past two weeks have I seen a case in which every vesicle examined was positive, and that was a very acute and extreme case involving both hands, with marked trichophytides on other parts of the body. Mitchell of Chicago, in his recent article on the subject in the Archives of Dermatology, ${ }^{3}$ states that the brownish, dried vesicles yield him the best results, and my experience agrees with this, especially in the older cases. Sometimes the desquamating collarettes left from this vesiculation will be positive. Crusting and exudative material is of no value, nor are the parakeratotic scales which are often seen.

(c) In the nail cases it is well to scrape the surface with a glass slide and from this cleaned surface to pare thin pieces with a knife. The scrapings with glass are beautifully thin and one is tempted to use them, but they are not of much value because the fungi do not grow in one stratum, and too thin scrapings may only show them in section, when they are unrecognizable.

Having taken the material to be examined, it is the best procedure to keep it between two glass slides whose contiguous surfaces have been flamed. These can be held by a rubber band and marked with a colored pencil for future identification. I believe the slides are superior to envelopes or paper containers since it is much easier to cut up and manipulate the scales in planting when they are on a glass surface; the scales often stick to the paper containers and it is difficult to remove them with as little handling as in the use of slides; and then, too, the paper containers are never sterile.

After the material is collected it may be kept almost indefinitely before planting, and a certain amount of delay is advantageous since it may eliminate many of the bacterial contaminations. An exception to this statement arises in the case of the examination and planting of fresh vesicles. 'These should be planted and examined immediately. The roofs of vesicles when dried are much more frequently negative than fresh ones from the same case.

For the microscopical examination of the material, use is made of the clearing effect of $\mathrm{KoH}$ or NaOH on the tissue cells, while the fungi are resistant, the result being that the fungi show as refractile bodies against a fairly clear background. The disadvantage in the use of KoII is that in a comparatively short time, depending on the strength used, the fungi are affected also, so that for permanent preparations some other clearing solution is necessary.

For rapid work a 40 per cent. aq. solution of KoII is used and the slide very gently heatcd. By this method scales may be examined in a few minutes. For slower clearing and more permanent preparations which will keep for about ten days one may use equal parts of a 30 per cent. solution of $\mathrm{KoH}$ and glycerine. This should be heated also, and scales are well eleared in one or two hours if not too thick. This is the method of choice unless permanent preparations are wanted.

For permanent preparations which will last for six months to a year, or perhaps slightly more, we use

$\begin{array}{ll}\text { Iuactic acid } & \text { 1. part } \\ \text { Phenol } & 1 \text { part } \\ \text { Glycerine } & \mathbf{2} \text { parts } \\ \text { Distilled water } & \mathbf{2} \text { parts }\end{array}$

This clears quickly and well, its only disadvantage being a tendency to the formation of very fine, long crystals which might deceive the beginner when searching for the fungi.

These permanent mounts have to be ringed with a cement, and by far the most satisfactory for this or any similar purpose is one made by Professor R. E. Schuh, Howard University, Washington, D. C.

Before clearing scales it is always wise to remove the fat by washing with ether. The KoH on a scale which contains much fat gives rise to many refractile globules which may be very deceptive and may make one think they are mycelial fragments. These globules, however, vary a great deal in size-much more so than mycelial fragments ever do. The $\mathrm{KoH}$ also affects many of the epithelial cells, so that they appear crowded with spores; an appearance which should not deceive the investigator, since these fungi do not penetrate the cells and do not form spores in the human host.

Having mounted and cleared the specimen, one searches with patience for the parasite, 
which should appear as a refractile septate strand-sometimes branching, and sometimes broken up in a chain of fragments. Often separate fragments or bodies resembling them are seen, but one should never make a positive diagnosis on one or more fragments unless they are found in chains. These fragments are commonly spoken of as spores, but most authorities agree that they are not true spores but fragments of the hypha or vegetative part of the fungus.

'Whe high dry lens of the microscope should be used, with much of the light cut off, and since the scales are usually quite thick one must examine all levels by focussing up and down. When expert, the fungi can frequently be picked up with the low power, which is a great saving of time, but in all cases, before pronouncing a case negative, several hours should be spent in careful search.

There are various staining methods which have been used to demonstrate the fungi in scales and hairs. Personally I do not use any of them for the ordinary clinical work. They take much more time than the methods just described, and are uncertain and rather unsatisfactory in their results. Practically their only advantage is in the making of permanent preparations, and even in the best preparations there is a certain amount of distortion, so that the picture is not the same as that of the fresh specimen.

All the fungi show two stages of growththe simple vegetative, and the form in which certain portions of the hyphae are differentiated for the purpose of reproduction. The primary reproductive body of the fungi is called a spore, and in the fungi it performs a function analogous to that of seeds in the higher plants. The vegetative forms are practically all alike-certainly not sufficiently different to allow identification of the different genera and species in the scales or hairs, so that the manner of producing spores or fruiting bodies is the chief means at present known for such identification. The reason for this short excursion into mycology lies in the fact that in the host the fungi show only their vegetative form, and in order to discover their manner of spore production and other distinguishing features they must be grown outside the host. This, then, is our reason for cultivating these organisms, to allow of their identification and classification, and to correlate if possible the various genera and species with definite clinical manifestations. Such work has not advanced very far. The mycological classification is still very hazy and the differences between many species are slight. One other mycological detail must be mentioned before considering the culture media. On the media containing sugars most of these fungi, after a varying length of time, take on the so-called pleomorphic form, which is a return to the vegetative manner of growth. 'lhis shows as a white, fluffy overgrowth of hyphac, which do not produce spores, and transplants from which always show this same pleomorphic growth, never the characteristies of the original eulture. On this account all cultures have to be grown on two media, one containing carbohydrate to show the fructification, and the other, without carbohydrate, to preserve the culture from the pleomorphic change.

All these fungi show different manners of growth on different media, and the following were devised by Sabouraud in Paris as international proof media, so that results in different countries might agree. Unfortunately the war interfered with the production of the French sugars used, so that work is now being done in this country to find what sugars can be used with the same result.

Sabouraud's media were as follows:
(a) Glucose Agar

$\begin{array}{lll}4 \% & \text { (b) } \begin{array}{l}\text { Multose } \\ 1 \%\end{array} \\ 1.5 \% & \text { Pgeptone }\end{array}$
$4 \%$
$1 \%$
$1.5 \%$
and (c) The conservation media
Peptone 3\%
Agar $\quad 1.5 \%$

The French glucose and maltose are no longer obtainable, but it has been found that for practically all purposes American pure dextrose (DifCo) will do. French peptone (Peptone Chassaing) is necessary in growing certain fungi on account of its acidity, and it is still available.

The ingredients are mixed as usual in making melia, and sterilized, care being taken not to overheat in the sterilization as this changes the sugars. Short fractional sterilization is the best method. For the first plantings of the original material slants in tubes are used. For further study of the growths obtained plants are made in Erlenmeyer flasks, which give more material and space for the fungi to show their characteristic manner of growth. Petri dishes may be used, but they do not give a sufficient depth of media, and contaminations are apt to creep in at the edges. The water of condensation which collects on the cover also interferes with inspection and photography of the growth.

In making the first plants the dried scales are cut up into very small pieces and planterl on the agar, about four pieces to a tube. At least 20 plants should be made in each case, but often as many as a hundred are necessary before getting a growth. The great obstacle to pure cultures is the presence of pus organisms and saprophytic moulds. Various methods have been used to inhibit these, such as flaming the scales, washing in alcohol, putting gentian violet $(1: 500,000)$ in the media, etc., 
but none of these are very satisfactory. Contaminations are the great bane of this work.

The cultures are grown at room temperature. After obtaining a growth it is transplanted to the three different media and its growth on each is compared. It is grown in hanging drop preparations of glueose bouillon, and direct mounts are made and mounted in glycerine for study. The authoritative work on this subject is by Sabouraud. ${ }^{4}$ Unfortunately our results do not always check up with his, partly because our media differ, and partly, also, because we probably have a different flora in this country.

This part of the subject should not be left without warning any who may be interested that the results, especially of the cultures, are distinctly discouraging compared with the amount of time and patience expended. Personally, after nearly two years' work I am unable to get growths in more than one out of four or five eases investigated-that is, of the eases in which the skin is affected. In practically every case of ringworm of the scalp the fungus ean be grown. The work is important, however; it offers practically a new field and can be carried on in spare moments and without the necessity of elaborate laboratory paraphernalia.

\section{TREATMISN'T.}

In marked contrast to tinea circinata, epidermophytosis is ordinarily very resistant to treatment, and it is difficult to explain why an organism which exists in the host only in its vegetative form should be able to resist for so long parasiticidal applications, or why it should so frequently recur in its original site long after all traces have apparently disappeared.

Probably the application most commonly used in this country is Whitfield's ointment. The formula for this as given in the last edition of Whitfield's book is:

$\begin{array}{lc}\text { Acid benzoici } & \text { gr. XXV } \\ \text { Acid sulicylici } & \text { gr. XV } \\ \text { l'araffini molii } & 3 \text { II } \\ \text { Ol. cocois nuciferne nd } & \text { J I }\end{array}$

Most dermatologists in this country use it somewhat stronger than this, even up to 12 per cent. and 18 per cent. of the salicylic and benzoic acids respectively. To a certain extent this ointment may be used as a therapeutic test, since dermatoses resembling epidermophytosis are quite apt to be made much worse by its use. A certain number of bona fide cases of the disease are, however, much irritated by Whitfield's ointment, so that the test is not decisive.

In France, chrysarobin in one per cent. strength is used more than in this country. Its drawbacks are its staining properties and its severe irritant action if not used with precaution. There is no doubt, however, that it

is a valuable application. It may be used in an ointment or painted on in liq. gutta percha.

Whitfield recommends for the resistant foot cases :

Chrysarobin

Ether sulphurici

Acetone
3 I

an 3 IV ss

painted on during the day, and the benzoic and salicylic acid ointment at night.

Iodin undoubtedly has stronger parasiticidal effect on these fungi in vitro than any of the other substances employed. When used clinically, however, it is far from being infallible. It is used as the tincture, painted on ; in ointments, 5 per cent., goose grease being the best excipient.

Dr. E. Wood Ruggles ${ }^{\mathfrak{b}}$ reports success from the use of this ointment:

$\begin{array}{lr}\text { Yinc: Oxide } & (\mathbf{0} \\ \text { Ung. picis licl. } & 1: 2 . \\ \text { Phenol } & 1 . \\ \text { Ung. Aq. Ros. } & 1.5 .\end{array}$

followed, after the eruption has well subsided, by

Tinct. Iodi.
Spir. Camph. an 28.

Other methods are $1: 5,000$ potassium permanganate soaks or hot fomentations for 15 minutes three times daily-combined with Whitfield's ointment. Saturated aqueous solution of picric acid painted on twice daily for a week. This should not be used continuously for longer than this on account of danger of absorption.

The x-ray has been used with some success and seems to act by modifying the skin so that it is no longer favorable to the growth of the fungus. The x-rays are not directly parasiticidal.

Ultra-violet ray therapy acts favorably in a certain number of cases, probably because of its desquamating effect.

1 Greenham, S. S., and klauder, J. V.: Yeast Jufections of the Skin: Ireport of Cases and of Stulies of the Cutaneons Yensts, Arch. Dermat. \& Syph., Vol. v, 1), 332, March, 1022.

Bloch, 13.: Anm. de termat. et syjh., Vol. 2, p, 1, Jan., 1021; ibid Vol. 2, p. 65, Feb., 1021.

"Mitichell, Jumes H.: Further Studies on Ringworms of the llunds and Jeet, Arch. Jermat. \& Syph., Vol. 5, 1) 174, Fel., 1022 .

- Sulouraud, R.: Les Teignes, Paris, 1910.

- Rugrles, F. Wood: Therapeutic Suggestions Regarding the Trentment of Affections of the IHancls and Foot, Arch. Dermat. \& Syph., Vol. v, p. 462, April, 1022.

\section{AUTOMOBILE NXHAUST GAS AS A HEALTH HAZARD.*}

By Yandelit: Henderson, Pir.D., New IIaven, Conn. Laboratory of Applied Physiologly, Yale Thiversity.

WITI every advance in industry and the arts there is liable to be associated some new physiological strain or health hazard. This has been

*A paper read before the IIarvard Medicnl Society, January 24, 1022, and before the American Climatological and Ólinical Asso: ciation, May, 1022 . 